\title{
Transplanted hematopoietic stem cells demonstrate impaired sarcoglycan expression after engraftment into cardiac and skeletal muscle
}

\author{
Karen A. Lapidos, ${ }^{1}$ Yiyin E. Chen, ${ }^{2}$ Judy U. Earley, ${ }^{2}$ Ahlke Heydemann, ${ }^{2}$ Jill M. Huber, ${ }^{2}$ \\ Marcia Chien, ${ }^{3}$ Averil Ma, ${ }^{3}$ and Elizabeth M. McNally2,4 \\ ${ }^{1}$ Department of Molecular Genetics and Cell Biology and 'Department of Medicine, University of Chicago, Chicago, Illinois, USA. \\ ${ }^{3}$ Department of Medicine, UCSF, San Francisco, California, USA. ${ }^{4}$ Department of Human Genetics, University of Chicago, Chicago, Illinois, USA.
}

\begin{abstract}
Pluripotent bone marrow-derived side population (BM-SP) stem cells have been shown to repopulate the hematopoietic system and to contribute to skeletal and cardiac muscle regeneration after transplantation. We tested BM-SP cells for their ability to regenerate heart and skeletal muscle using a model of cardiomyopathy and muscular dystrophy that lacks $\delta$-sarcoglycan. The absence of $\delta$-sarcoglycan produces microinfarcts in heart and skeletal muscle that should recruit regenerative stem cells. Additionally, sarcoglycan expression after transplantation should mark successful stem cell maturation into cardiac and skeletal muscle lineages. BM-SP cells from normal male mice were transplanted into female $\delta$-sarcoglycan-null mice. We detected engraftment of donor-derived stem cells into skeletal muscle, with the majority of donor-derived cells incorporated within myofibers. In the heart, donor-derived nuclei were detected inside cardiomyocytes. Skeletal muscle myofibers containing donor-derived nuclei generally failed to express sarcoglycan, with only 2 sarcoglycan-positive fibers detected in the quadriceps muscle from all 14 mice analyzed. Moreover, all cardiomyocytes with donor-derived nuclei were sarcoglycan-negative. The absence of sarcoglycan expression in cardiomyocytes and skeletal myofibers after transplantation indicates impaired differentiation and/or maturation of bone marrow-derived stem cells. The inability of BM-SP cells to express this protein severely limits their utility for cardiac and skeletal muscle regeneration.
\end{abstract}

\section{Introduction}

Stem cell therapy for skeletal and cardiac disease is an attractive long-term strategy for the delivery of normal, pluripotent cells to necrotic regions for the purpose of tissue regeneration. While a myriad of factors will play a role in the final engraftment outcome of any type of stem cells, the identification of the ideal stem cell population for this application remains elusive. Bone marrow is an attractive source of stem cells, since it is thought to contain stem cell pools that can repair tissues as diverse as skeletal muscle, heart, epithelium, liver, and brain $(1,2)$. The nonadherent component of bone marrow is reported to contain stem cells with myogenic potential (3). Transplantation of whole bone marrow, or even a single CD45-positive cell, resulted in donor cell fusion with preexisting myofibers to promote regeneration $(4,5)$.

Side population (SP) cells are a stem cell variant isolated by their selective ability to rapidly efflux the DNA-binding dye Hoechst 33342. Bone marrow-derived SP (BM-SP) cells represent only $0.01-0.05 \%$ of whole bone marrow and were originally identified as HSCs (6). The potential of BM-SP cells to regenerate skeletal muscle was noted after systemic transplantation into irradiated

Nonstandard abbreviations used: BM-SP, bone marrow-derived side population; IFM, immunofluorescence microscopy; Sgct ${ }^{-/}$, $\delta$-sarcoglycan-null (mice); SP, side population.

Conflict of interest: The authors have declared that no conflict of interest exists.

Citation for this article: J. Clin. Invest. 114:1577-1585 (2004).

doi:10.1172/JCI200423071. dystrophin-mutant $m d x$ mice and confirmed with restoration of dystrophin expression (7). The contribution of BM-SP cells to cardiac regeneration was documented in lethally irradiated mice transplanted with a refined bone marrow SP, characterized as $\mathrm{CD} 34-/$ low $/ \mathrm{c}-\mathrm{kit}^{+} / \mathrm{Sca}-1^{+}$, and then subjected to myocardial infarction (8). In this model, donor-derived cells were found in infarcted hearts, and differentiation was documented using colocalization with endogenous host myocyte markers. SP cells have now been isolated from skin, mammary epithelium, and heart; these stem cells have been shown to harbor regenerative potential using varying modes of transplantation, including systemic delivery and direct organ injection (9-11). Although each SP shares the ability to rapidly efflux Hoechst 33342 and shows overlap of cell surface antigens such as Sca-1, it is unclear whether all tissue-derived SP cells share a common progenitor cell.

Collectively, these transplantation studies and others $(4,12)$ indicate that stem cells successfully home to skeletal and cardiac muscle. In many transplantation studies, engraftment has been detected by the presence of broadly expressed, donor cell markers such as GFP, LacZ, or the Y chromosome. These markers lack tissue specificity, as they are expressed in all donor cells. To accommodate the nonspecificity of these markers, colocalization with endogenous markers was used to document differentiation into particular lineages. However, the endogenous markers typically cannot be used to distinguish between donor and recipient cells. To provide a specific marker of stem cell differentiation, recent studies using a donor-derived, tissue-specific marker reported 
A
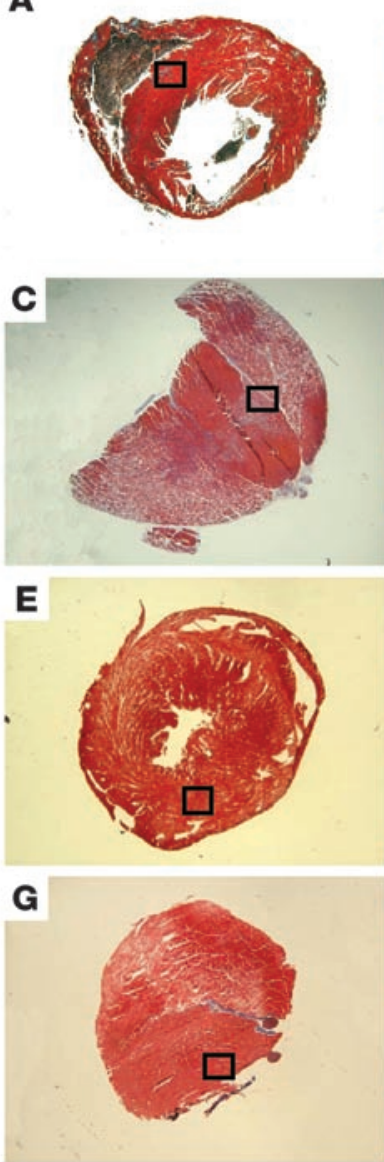

Figure 1

$S g \mathrm{~cd}^{-/-}$mice develop focal degeneration in heart and skeletal muscle that leads to cardiomyopathy and muscular dystrophy. (A-D) The focal degeneration in $\delta$-sarcoglycan-null myocardium (A and $\mathbf{B})$ and striated muscle (C and D) can be seen with Masson trichrome staining. $(\mathbf{E}-\mathbf{H})$ Also shown is normal cardiac ( $\mathbf{E}$ and $\mathbf{F})$ and skeletal muscle ( $\mathbf{G}$ and $\mathbf{H})$. $\mathbf{B}, \mathbf{D}, \mathbf{F}$, and $\mathbf{H}$ show higher-magnification views of the boxed regions in $\mathbf{A}, \mathbf{C}, \mathbf{E}$, and $\mathbf{G}$, respectively.

that bone marrow-derived stem cells do not acquire cardiomyocyte status after transplantation (13). In this model, expression of a reporter marker, GFP, was driven by a transgene containing the cardiomyocyte-specific $\alpha$-myosin heavy chain promoter. In contrast, the use of a recipient host that is deficient for a donorderived, endogenous marker is ideal for establishing the differentiation potential of the stem cell pool, since the detection of the marker indicates that the cell is both myogenic and donor-derived. The detection of dystrophin in $m d x$ recipient mice should fulfill these criteria and has been exploited in transplantation studies (14). However, not all dystrophin-positive fibers in this setting are donor-derived. The untransplanted, $m d x$ mouse exhibits "revertant" fibers that express dystrophin due to exon skipping. Such dystrophin-positive, revertant myofibers may be as high as $5 \%$ of total myofibers in $m d x$ mice.

Members of the sarcoglycan complex are ideal endogenous markers for stem cell transplantation. The sarcoglycans form a membrane-associated complex that associates with dystrophin in mature striated muscle. The loss of $\delta$-sarcoglycan produces pro- gressive cardiomyopathy and muscular dystrophy in humans and mice (15-17). Intrinsic focal damage in $\delta$-sarcoglycan-null ( $\left(\mathrm{Scd}^{-/}\right)$ mice should serve as a potent recruiter of stem cells for cardiomyocyte and myofiber regeneration. Sgcd ${ }^{-/-}$mice were generated through homologous recombination and express no $\delta$-sarcoglycan protein and have no revertant fibers (15). In addition, the absence of $\delta$-sarcoglycan leads to the loss of all other sarcoglycans at the membrane, providing a completely null background for the detection of sarcoglycan-positive cells in transplant recipients. The presence of sarcoglycan in a sarcoglycan-null recipient after transplantation provides a stringent condition for assessing the therapeutic value of stem cells, since sarcoglycan will only be expressed if the donor-derived cell has matured to a myocyte fate.

We transplanted BM-SP cells from normal male mice into female $S g c d^{-/}$recipients to evaluate whether focal damage in $\delta$-sarcoglycan-null muscle would recruit stem cells for regeneration. We found that donor-derived cells readily engrafted into $\delta$-sarcoglycan-null myofibers and cardiomyocytes. Unexpectedly, we found that the donor-derived cells were unable to restore sarcoglycan expression and the sarcoglycan complex. The inability of BM-SP cells to express $\delta$-sarcoglycan after transplantation into striated muscle demonstrates that BM-SP cells are limited in their differentiation potential. Sarcoglycan expression is required for normal heart and skeletal muscle function, so these studies suggest that BM-SP stem cells have limited potential for cardiac and skeletal muscle regeneration.

\section{Results}

A null mutation in $\delta$-sarcoglycan leads to focal degeneration in heart and skeletal muscle. Sgcd-/- mice develop muscular dystrophy and cardiomyopathy (15). In $S g c d^{-/-}$mice and in the $\delta$-sarcoglycanmutant Syrian cardiomyopathic hamster, the degenerative process in both skeletal and cardiac muscle is focal in nature and has been described as "microinfarction" (18). That is, regions of degeneration are seen scattered throughout striated muscle and are accompanied by abnormal vascular reactivity. We hypothesized that the focal nature of degeneration in sarcoglycan-null mice would serve to recruit stem cells, since stem cells have been shown to home to damaged striated muscle (19-21). Figure 1 shows sections from cardiac and skeletal $S g c d^{-/}$muscle that highlight the focal nature of degeneration, seen as the blue stain of Masson trichrome (Figure 1, A-D).

$B M$-SP cells engraft into skeletal muscle but do not restore sarcoglycan expression. Lineage-negative BM-SP cells were isolated from male mice and injected i.v. into female $\mathrm{Sgcd} /-$ mice to assess their ability to engraft and differentiate into skeletal muscle. SP cell preparations were verapamil-sensitive and consistently enriched for Sca-1 positivity (Figure 2). Approximately 77\% of the donor BM-SP cells were positive for this stem cell marker, consistent with other reports $(6,7)$ (Figure 2). BM-SP cells were 40-50\% c-kit-positive. Three weeks or 3 months after transplantation, sections from quadriceps muscles were evaluated using immunofluorescence microscopy (IFM) with an antibody to $\gamma$-sarcoglycan followed by FISH with a Y chromosome-specific probe (Y-1). We examined sections for $\gamma$-sarcoglycan because restoration of $\delta$-sarcoglycan in $\mathrm{Sgcd}^{-/-}$mice, through transgenesis or adenoviral transduction (22-25), leads to expression of $\gamma$-sarcoglycan and reflects a reconstituted and functional sarcoglycan complex (Figure 3, A-C). FISH with the Y-1 probe in normal male muscle detects approximately $70-80 \%$ of all nuclei. Using this method, we detected Y chromosome-positive cells in 
A
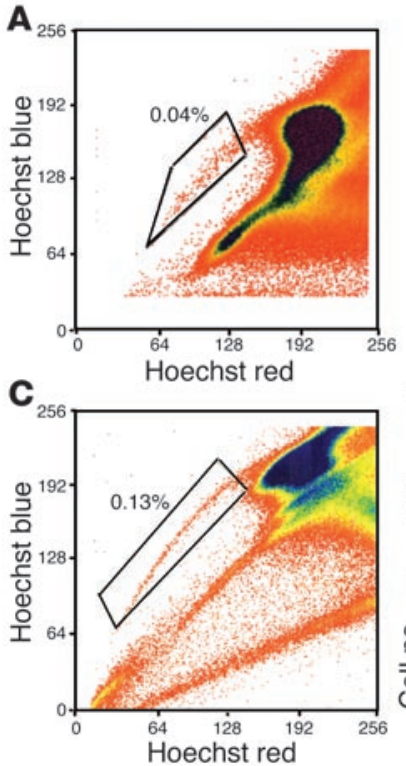

D
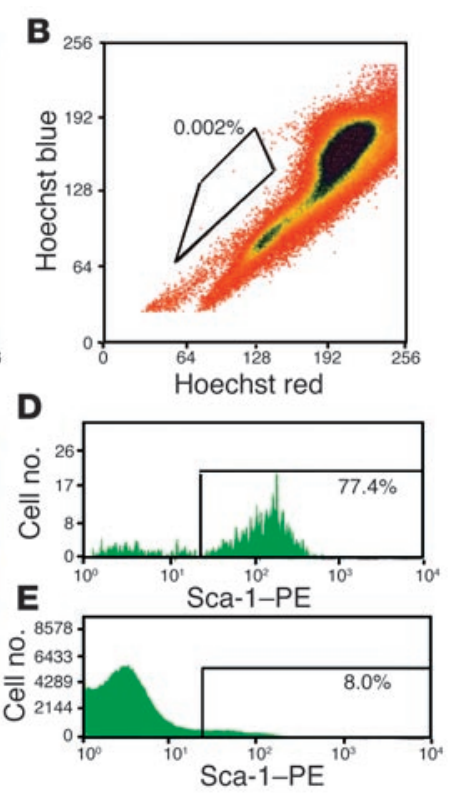

every $S g c d^{-/}$female recipient mouse. In all recipients, Y-1-positive cells were found in between myofibers or within myofibers, as central or peripheral nuclei (Figure 3E). However, despite engraftment of Y chromosome-positive nuclei into muscle and to myofibers, expression of $\gamma$-sarcoglycan was generally absent (Figure 3F).

We examined quadriceps muscle from a total of 14 recipient mice and therefore examined approximately 10,000 myofibers from each recipient animal. Of 14 quadriceps muscles examined, we detected only 2 myofibers that stained positively for $\gamma$-sarcoglycan (Figure 3G). These myofibers were also positive for $\delta$-sarcoglycan immunoreactivity consistent with restoration of the sarcoglycan complex (Figure $3 \mathrm{H})$. For 1 of the 2 myofibers, $\gamma$-sarcoglycan staining extended for more than $300 \mu \mathrm{m}$ along the length of the myofiber. Interestingly, this myofiber showed neither host-nor donor-derived nuclei in these sections. As every tenth tissue section was collected and examined, the donor-derived nucleus responsible for sarcoglycan expression most likely resided in between sampled sections.

\section{Figure 3}

BM-SP cells engraft into skeletal muscle but have limited sarcoglycan expression. Normal male quadriceps $(\mathbf{A}-\mathbf{C})$ and female $\mathrm{Sgcd}^{-/-}$recipient quadriceps muscle sections (D-F) were stained for $\gamma$-sarcoglycan immunoreactivity ( $\mathbf{A}$ and $\mathbf{D}$, red) and then subjected to FISH with a $Y$ chromosome-specific probe, $\mathrm{Y}-1$ ( $\mathbf{B}$ and $\mathbf{E}$, green). The merged images along with DAPI staining (blue) in $\mathbf{C}$ and $\mathbf{F}$ show nuclear localization of the $\mathrm{Y}-1$ signals. Arrows in $\mathbf{D}-\mathbf{F}$ indicate $\mathrm{Y}$-1-positive cells engrafted between myofibers. The arrowheads in $\mathbf{D}-\mathbf{F}$ show a $\mathrm{Y}$-1-positive nucleus inside a myofiber. Despite engraftment, sarcoglycan expression was not restored. (G) A single $\gamma$-sarcoglycan-positive cell detected in $\delta$-sarcoglycan-null quadriceps after transplantation with BM-SP cells. (H) A serial section of the same donor-derived cell also expresses $\delta$-sarcoglycan. (I) Immunostaining of a transplant-recipient muscle with dystrophin and concomitant FISH with the Y-1 probe to detect donor nuclei (dystrophin, red; Y-1, green; DAPI, blue). Arrowheads in I indicate $3 \mathrm{Y}$ chromosome signals in the central or peripheral nucleus position. Scale bars: $50 \mu \mathrm{m}$.

\section{Figure 2}

Hoechst dye exclusion technique isolates SP cells. Whole bone marrow was incubated with Hoechst 33342 with or without verapamil and then subjected to FACS analysis. (A and B) Typical FACS profiles. A shows that the SP cells represent approximately $0.04 \%$ of whole bone marrow. B indicates the expected verapamil sensitivity of Hoechst dye. (C-E) Whole bone marrow was incubated with Hoechst dye and then stained with a PE-conjugated Sca-1 antibody and a mouse lineage antibody cocktail. Lineage-positive cells were magnetically depleted from whole bone marrow, and lineage-negative cells were subjected to FACS analysis. (C) A FACS profile. The boxed region in $\mathbf{C}$ indicates that the SP represents $0.13 \%$ of lineage-negative cells. ( $D$ and $E$ ) The results of staining these cells with Sca- 1 . SP cells are $77 \%$ Sca-1-positive, and lineage-negative cells are $8 \%$ Sca-1-positive.

Engraftment of BM-SP cells into skeletal myofibers of $\delta$-sarcoglycan recipients. Irradiation is lethal to HSCs and muscle satellite cells and is thought to create a niche that allows for increased engraftment of exogenous stem cells $(6,26,27)$. Sublethal irradiation significantly increased total engraftment in skeletal muscle, more than fivefold (97.6 versus 18.6 Y-1-positive nuclei per square millimeter) (Figure 4A). Increasing the length of time after transplantation had no significant effect on the number of Y-1-positive nuclei per square millimeter. We calculated the percentage of total nuclei that were Y-1-positive and, by this method, also found that irradiation increased engraftment from $0.82 \%$ to $4.2 \%$ (Table 1). Mice sacrificed 3 months after transplantation showed an engraftment level of $5.5 \%$, similar to the engraftment level observed in the 3-week irradiated group.

Skeletal muscle regenerates through the fusion of a myogenic stem cell with a damaged myofiber. To correct the genetic and cellular defects found in $\delta$-sarcoglycan-null skeletal myofibers, stem cells must engraft inside the myofibers. Thus, the percentage of Y-1-positive nuclei that localized within myofibers was quantified. Each Y-1-positive nucleus was scored for its position relative to the myofiber plasma membrane using host-derived dystrophin as a sarcolemmal marker (Figure 3I). Nonirradiated recipients had the highest percentage of Y-1-positive nuclei within myofibers (69.4\%) (Table 1). In contrast, over $90 \%$ of the $\mathrm{Y}-1$-positive nuclei in the irradiated recipients were in the
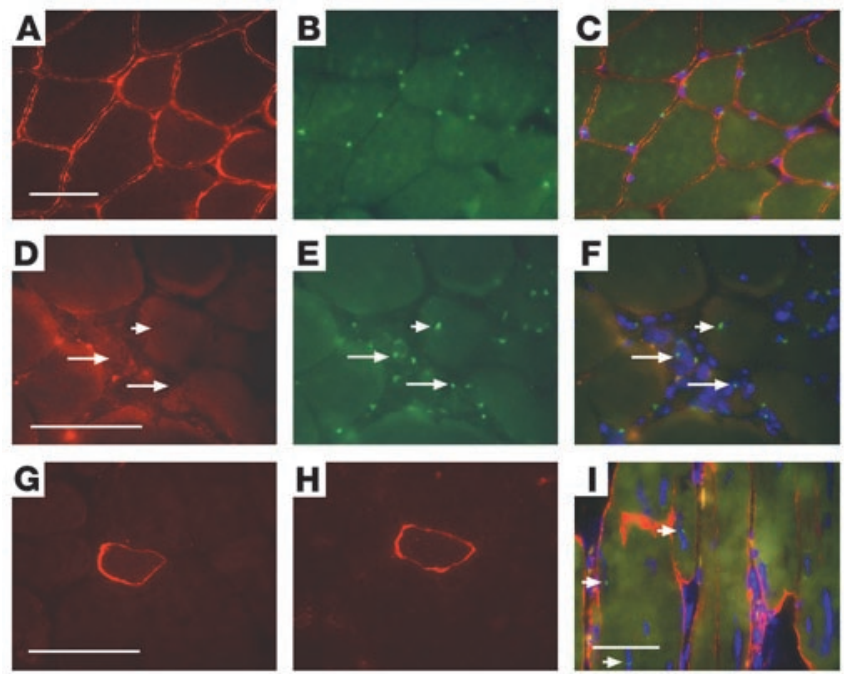


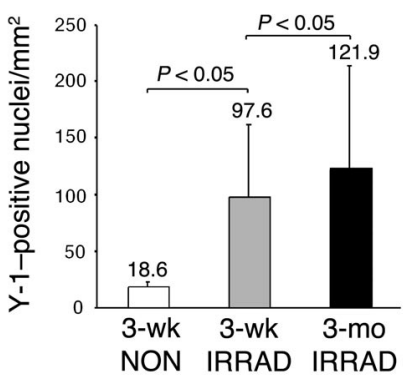

Figure 4

Irradiation increases total engraftment into skeletal muscle. The number of Y-1-positive nuclei per square millimeter was quantified in recipient mice irradiated before transplantation, or not irradiated, and sacrificed 3 weeks after transplantation (3-wk IRRAD and 3-wk NON, respectively; $n=5$ of each). The number of Y-1-positive nuclei per square millimeter was also quantified in recipient mice irradiated before transplantation and sacrificed 3 months after transplantation (3-mo IRRAD; $n=4$ ). The 3-week irradiated group showed a significantly higher number of $\mathrm{Y}$-1-positive nuclei per square millimeter compared with the 3-week nonirradiated group (Kruskal-Wallis test followed by Dunn's multiple-comparison test, $P<0.05)$.

nonmyofiber position with only $7 \%$ and $2 \%$ of the nuclei directly incorporated in myofibers in the 3-week and 3-month irradiated groups, respectively. Therefore, while irradiation increased overall engraftment, the number of donor-derived myofiber nuclei was equivalent in irradiated versus nonirradiated recipients. However, despite significant engraftment of donor-derived cells directly into myofibers, these nuclei were unable to express sarcoglycan.

$B M-S P$ cells engraft into the myocardium but do not restore sarcoglycan expression. We next examined the potential of BM-SP cells to engraft and differentiate into cardiomyocytes in the sarcoglycannull model of cardiomyopathy. Sections from female recipient Sgcd ${ }^{-/}$hearts were examined with sarcoglycan staining followed by FISH with the Y-1 probe (Figure 5A). All transplanted mice showed the presence of Y-1-positive nuclei. However, no $\gamma$-sarcoglycan was detected in over 400 sections examined from 14 recipient hearts. Irradiation significantly increased the engraftment of Y-1-positive nuclei into cardiac muscle, 50-fold (Figure 6). Increasing the length of time after transplantation and before sacrifice also significantly increased engraftment, 12 -fold, compared with that in the 3 -week irradiated group (1.68 versus 20.5 Y-1-positive nuclei per square millimeter). The 3 -week nonirradiated group showed only $0.0008 \%$ of the nuclei to be Y-1-positive, whereas irradiation increased engraftment more than 50 -fold, to $0.043 \%$ (Table 2). Sacrificing the mice 3 months after transplantation also significantly increased engraftment, to $0.53 \%$, which reflects a 12 -fold increase compared with engraftment in the 3 -week irradiated mice.

The distribution of Y-1-positive nuclei in recipient hearts was determined by costaining with dystrophin. Over 2,000 Y-1-positive nuclei were scored. As in skeletal muscle, nonirradiated recipients showed the highest percentage of incorporation into cardiomyocytes (64.7\%), despite the lowest total number of Y-1-positive cells (Table 2). As in skeletal muscle, over $90 \%$ of the Y-1-positive nuclei detected in the irradiated recipients were in the nonmyocyte position. Only $7 \%$ and $2 \%$ of the Y-1-positive nuclei were incorporated into myocytes in the 3-week and 3-month irradiated groups, respectively. Thus, while each group showed incorporation of Y-1-positive nuclei into cardiomyocytes, donor-derived cells were unable to express sarcoglycan. Cardiomyocytes containing Y-1positive nuclei were indistinguishable from other cardiomyocytes in size and shape. Cardiomyocytes containing 2 nuclei per cell were seen in which 1 nucleus was Y-1-positive (Figure 5B).

Irradiation supports hematopoietic differentiation of BM-SP cells. Irradiation significantly increased total engraftment of BM-SP cells into skeletal and cardiac muscle. However, most of this engraftment was not directly into myocytes but rather was into the interstitial cells; this reflects the known inflammatory infiltrate that accompanies cardiomyopathy and muscular dystrophy (28). To examine the potential of BM-SP cells to differentiate into hematopoietic cells, tissue sections were stained with the lymphocyte marker CD45 and processed for FISH to detect the Y chromosome. The skeletal muscle of each irradiated animal $(n=9)$ revealed numerous Y-1-positive cells localized alone or in clusters that were CD45-positive, consistent with differentiation along the hematopoietic pathway (Figure 7, A and B). In addition, CD45-positive, donor-derived cells were detected in the hearts of irradiated mice (Figure 7, C and D).

Primary myoblast transplantation restores sarcoglycan expression. To determine whether sarcoglycan-null myofibers can accommodate wild-type stem cell regeneration, primary myoblasts were isolated and cultured from normal, neonatal male mice and transplanted into sublethally irradiated female $\operatorname{Sgcd}^{-/-}$mice $(n=2)$. The cells were injected directly into the tibialis anterior muscle, since the size of this muscle is convenient for injection and analysis. Myofibers positive for $\gamma$-sarcoglycan were readily detected, with the highest numbers of sarcoglycan-positive cells closest to the injection site. Normal skeletal muscle sections stained for $\delta$-, $\gamma$-, and $\beta$-sarcoglycan are shown (Figure 8, top panels) along with untransplanted $\mathrm{Sgcd}^{-/}$muscle (Figure 8, middle panels). Serial sections in the myoblast transplant recipient show a cluster of cells positive for $\delta$-, $\gamma$-, and $\beta$-sarcoglycan, confirming that the sarcoglycan complex can be restored through transplantation in $\mathrm{Sgcd} \mathrm{C}^{-/}$mice. Immunofluorescence followed by FISH with the Y-1 probe showed that $Y$ chromosome-positive nuclei were incorporated into $\gamma$-sarcoglycan-positive cells (data not shown). We similarly tested whether direct injection of BM-SP cells would support sarcoglycan expression after engraftment. For these experiments, 7,000 BM-SP cells were directly injected into the tibialis anterior in a manner identical to that of myoblast injections ( $n=2$ recipient mice). Four-andone-half weeks after injection, mice were sacrificed, and every third section throughout the entire tibialis anterior was examined for

\section{Table 1}

Engraftment of donor-derived nuclei in skeletal muscle

$\begin{array}{lccc} & \text { 3-wk NON } & \text { 3-wk IRRAD } & \text { 3-mo IRRAD } \\ \text { Engraftment (\%) } & 0.82 & 4.2 & 5.5 \\ \text { Nonmyofiber (\%) } & 30.6 & 92.4 & 98.1 \\ \text { Myofiber (\%) } & 69.4 & 7.6 & 1.9 \\ \text { Peripheral (\%) } & 27.9 & 28.9 & 73.7 \\ \text { Central (\%) } & 72.1 & 71.1 & 26.3\end{array}$

Donor cell engraftment was calculated as the percentage of total nuclei that were $\mathrm{Y}$-1-positive for each group. Also shown for each group is the percentage of $Y$-1-positive nuclei that engrafted between myofibers in a nonmyofiber position or directly into myofibers. Dystrophin immunostaining was performed on these sections to outline myofibers. Of $\mathrm{Y}-1-$ positive nuclei found within myofibers, the percentages in the central or peripheral positions are indicated. 

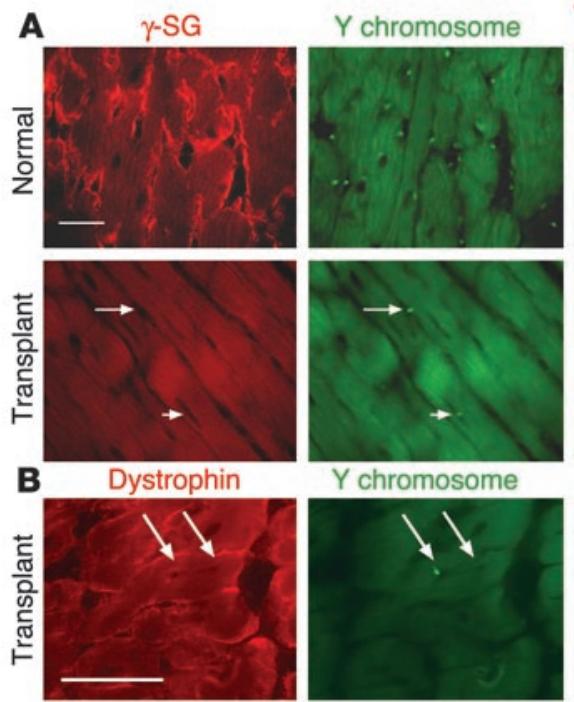

Y chromosome
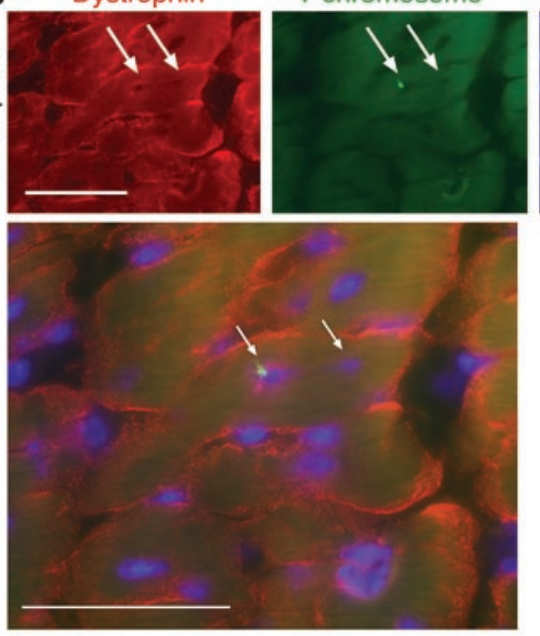

$\gamma$-sarcoglycan expression. No $\gamma$-sarcoglycan expression was detected (data not shown).

\section{Discussion}

A number of studies have documented that bone marrowderived stem cells regenerate cardiomyocytes and skeletal myofibers. For example, transplantation with whole bone marrow (14) or the more refined SP (7) has been reported to repair both skeletal and cardiac muscle in dystrophin-deficient $m d x$ mice (14). In the heart, whole bone marrow and refined bone marrow preparations have shown a high level of engraftment of donor-derived cardiomyocytes adjacent to infarct regions and functional improvement in the heart (29). For cardiomyocytes, it has recently been suggested that these stem cells do not have the ability to differentiate into cardiomyocytes and instead may be the product of stem cell fusion to mature cardiomyocytes (13, 30). Autopsy evaluation of human hearts after sex-mismatched bone marrow transplantation has been controversial with respect to the ability of donor-derived cells to differentiate into mature cardiomyocytes (31-34). Yet in humans, autologous bone marrow progenitor transplantation after acute myocardial infarction or in chronic heart failure has produced evidence of functional improvement (35-37). Because of these results, despite confusion as to their differentiation status, bone marrow-derived stem cells are being tested in human subjects with myocardial infarction, and the initial results have been promising (38).

We developed a direct approach to investigate the ability of BM-SP stem cells to differentiate into skeletal and cardiac muscle

\section{Figure 5}

BM-SP cells engraft into myocardium but fail to express sarcoglycan. (A) Sections from a normal male heart (top panels) and a transplanted female $\delta$-sarcoglycan-null heart (bottom panels) were stained for $\gamma$-sarcoglycan ( $\gamma$-SG) and then subjected to FISH with the Y-1 probe. The left panels show $\gamma$-sarcoglycan staining in red, the middle panels show $\mathrm{Y}-1$ in green, and the right panels show the merged image with DAPI in blue. Y chromosome-positive cells engrafted in the recipient myocardium between (arrow) or inside (arrowhead) cardiomyocytes but failed to restore sarcoglycan expression. YChr, Y chromosome. Scale bar: $50 \mu \mathrm{m}$. (B) Cross section through a cardiomyocyte containing 2 nuclei (arrows), where 1 is donor-derived. Cardiomyocytes are outlined with dystrophin (red), and the Y-1 probe (green) is found in the left nucleus but not the right. Scale bars: $50 \mu \mathrm{m}$.

in a mouse model of cardiomyopathy and muscular dystrophy. We selected BM-SP stem cells as donor cells because previous reports suggested that SP cells are pluripotent with the ability to contribute to both skeletal and cardiac muscle regeneration $(7,8)$. In our experiments, we readily detected the presence of donor-derived nuclei within myofibers and cardiomyocytes in sarcoglycan-null recipient mice. We detected a level of engraftment similar to what has been previously reported $(7,8)$, and a level that is much greater than the number of cells injected for each transplant. However, engraftment, as determined by the presence of a Y chromosomepositive nucleus, was not associated with the expected expression of sarcoglycan at the plasma membrane. The absence of sarcoglycan expression from wild-type donor-derived cells indicates impaired maturation of donor-derived nuclei. Additionally, the absence of sarcoglycan expression is associated with degeneration in cardiac and skeletal muscle. Therefore, the regenerative potential of BM-SP cells, as they do not express sarcoglycan to any significant degree, is highly limited.

SP cells have the drawback that they are heterogeneous and are not a clonal population. That said, BM-SP cells have been documented to possess considerable regenerative potential for heart and skeletal muscle. The BM-SP cells that we isolated are comparable to what others have reported $(6,7)$. In our hands, BM-SP cells were consistently Sca-1-positive and supported hematopoietic differentiation in sublethally irradiated mice. We also performed whole bone marrow transplantation experiments $(n=2)$ with a result similar to that seen with BM-SP cells and were unable to detect sarcoglycan expression in recipient heart and skeletal muscle (data not shown). As in competitive repopulation assays (6), our data suggest that irradiation provides a niche for BM-SP cell engraftment, proliferation, and hematopoietic differentiation. The numbers of donor-derived nuclei in each muscle exceeded the number of cells used in each transplant, demonstrating the highly proliferative potential of the transplanted stem cells.

The general failure of sarcoglycan expression by BM-SP cells after transplantation stands in contrast to what has previously been reported. Notably, it has been shown that bone marrow-derived cells restore dystrophin expression in the skeletal muscle of $m d x$ mice $(7,14)$. However, prior studies lacked the ability to detect whether the donor-derived nucleus was driving expression of dystrophin because of the presence of revertant fibers that produce host-derived dystrophin expression. In the present study, we used sarcoglycan expression as an endogenous marker of myofiber and cardiomyocyte determination and found that donor-derived nuclei did not achieve this standard. Myofibers and cardiomyocytes with 


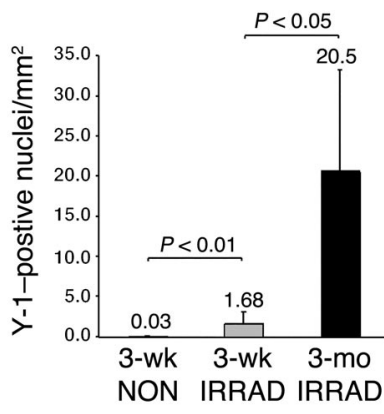

\section{Figure 6}

Irradiation and a longer time interval after transplantation increase overall engraftment into the myocardium. The number of $\mathrm{Y}$-1-positive nuclei per square millimeter was quantified for mice preirradiated or not preirradiated and sacrificed 3 weeks after transplantation (3-wk IRRAD and 3-wk NON, respectively; $n=5$ of each). The number of Y-1-positive nuclei per square millimeter was quantified for mice preirradiated and sacrificed 3 months after transplantation (3-mo IRRAD; $n=4$ ). A significantly higher number of $Y$-1-positive nuclei per square millimeter was detected in the 3-week irradiated group versus the 3-week nonirradiated group (Mann-Whitney test, $P<0.01$ ). The 3-month irradiated group showed a significantly increased number of $Y-1-$ positive nuclei per square millimeter compared with the 3-week irradiated group (Mann-Whitney test, $P<0.05$ ).

donor-derived nuclei continue to express myogenic markers, such as dystrophin, but it is likely that host nuclear expression is responsible for this expression. In our experiments, it is unclear to what degree donor-derived nuclei have entered into the myogenic program. For example, dystrophin and sarcoglycan are expressed late in myotube differentiation in cell culture models (39), while MyoD and desmin serve as early markers of myoblast status. Because sarcoglycan is a late marker of myogenic specification, it is possible that stem cell-derived nuclei have partially differentiated into a myocyte lineage. However, studies to determine whether donor-derived nuclei express a myogenic program are hampered by the robust expression of host myogenic genes and the multinucleate nature of myotubes and the binucleate nature of cardiomyocytes. As each cell contains nuclei from both donor and recipient, and contents of nuclei may be imported into donor-derived nuclei, the only reliable marker of muscle differentiation in our experiments is sarcoglycan. Sarcoglycan expression from its genomic promoter is a native marker of differentiation and, as such, may be a more reliable marker than transgene-driven markers of cellular determination that may demonstrate unusual tissue specificity or levels of expression.

We confirmed that sarcoglycan, as a cell surface complex, is not required on mature myotubes or cardiomyocytes for the successful engraftment of stem cells. $\delta$-Sarcoglycan-null myofibers accommodated myoblast engraftment and resulted in the expression of the sarcoglycan complex. In addition, recent studies using fetal stem cells from the dorsal aorta showed effective engraftment and restoration of the sarcoglycan complex in mice lacking $\alpha$-sarcoglycan (40). Thus, sarcoglycan-deficient muscle is capable of regeneration through transplantation. We contend that our stringent criterion of detecting sarcoglycan expression in $\mathrm{Sgcd}^{-/-}$recipient mice accurately reflects stem cell contribution to tissue regeneration. We conclude that BM-SP cells readily home to damaged skeletal and cardiac muscle but that donor-derived nuclei are impaired in their ability to express sarcoglycan.
Since the cardiac and skeletal muscle of both irradiated and nonirradiated mice contained similar absolute numbers of donorderived myonuclei, our experiments suggest that hematopoietic engraftment is not required for myocyte differentiation. In addition, while irradiation increased the total number of donorderived cells that engrafted into these tissues, it did not provide a selective advantage for sarcoglycan expression, since only 2 sarcoglycan-positive fibers were detected in a total of 14 mice analyzed. Collectively, our data caution against clinical studies in which irradiation and bone marrow transplantation are used for the treatment of muscular dystrophy and cardiomyopathy. In support of this caution, Gussoni et al. reported the presence of donor-derived nuclei within the myofibers of a Becker muscular dystrophy patient who had received a sex-mismatched bone marrow transplant 12 years earlier (41). In that work, it was ultimately demonstrated that dystrophin near donor cells was host-derived. In addition, the general plasticity of bone marrow has been challenged recently (42-45).

Cellular fusion, as opposed to de novo differentiation, may account for the apparent maturation impairment. In support of this, recent reports have suggested that the fusion of bone marrowderived cells is responsible for the observed "transdifferentiation" of stem cells. Using the Cre/lox system, bone marrow cells were shown to fuse with Purkinje neurons, hepatocytes, and cardiomyocytes undergoing de novo lineage-specific differentiation (46). In a study using genetically tagged cells, fusion also has been estimated to account for nearly half of engraftment and differentiation into cardiomyocytes in infarcted myocardium (47). In contrast to cardiac muscle regeneration, skeletal muscle regeneration normally relies on fusion between an immature cell and a preexisting myofiber. The absence of sarcoglycan expression after bone marrow stem cell transplantation suggests that fusion as it occurs with bone marrow-derived stem cells is quite distinct from normal myoblast fusion.

Interestingly, transplantation of bone marrow-derived stem cells has been associated with improved cardiac function (29). Mechanisms other than cardiomyocyte growth may contribute to functional improvement. For example, enhanced angiogenesis and growth factor recruitment are both likely to accompany stem cell transplantation and would improve cardiac function. As an alternative to bone marrow-derived cells, tissue-specific stem cell types should be considered for cell-mediated therapy of skeletal and cardiac muscle. For example, Sca-1-positive progenitors isolated from the hearts of adult mice (47) or muscle-derived stem cells may offer improved therapeutic efficacy (48). Fetal cells such as the vessel-associated mesangioblasts also show promise for significant muscle regeneration (40). Priming of transplant recipi-

\section{Table 2}

Engraftment of donor-derived nuclei in cardiac muscle

\begin{tabular}{lccc} 
& 3-wk NON & 3-wk IRRAD & 3-mo IRRAD \\
Engraftment (\%) & 0.0008 & 0.043 & 0.53 \\
Noncardiomyocyte (\%) & 35.3 & 92.9 & 97.6 \\
Cardiomyocyte (\%) & 64.7 & 7.15 & 2.38 \\
\hline
\end{tabular}

Engraftment was determined by quantifying the percentage of total nuclei that were $\mathrm{Y}$ chromosome-positive for each group. Also shown are percentages of engraftment into the noncardiomyocyte and cardiomyocyte position as determined after dystrophin immunostaining. 

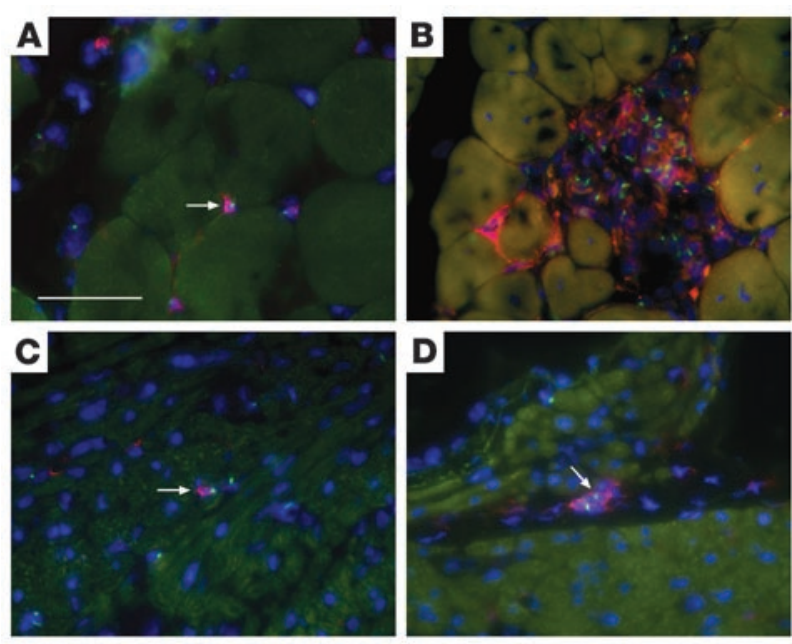

Figure 7

BM-SP cells in between myocytes express CD45 as a marker of hematopoietic differentiation. Heart and quadriceps sections were processed for IFM with an antibody to CD45 followed by FISH with the Y-1 probe. $Y$ chromosome hybridization signals are shown in green, CD45 is shown in red, and DAPI is shown in blue. Y chromosome-positive cells that are CD45-positive are readily detected in between skeletal myofibers as isolated cells (arrow, A) or in large clusters (B). CD45positive donor-derived cells are also found in between cardiomyocytes in the heart of recipient mice after transplantation (arrows, C and D). Scale bar: $50 \mu \mathrm{m}$.

ents with growth or other mobilizing factors may also improve engraftment (12), and these factors may also improve differentiation and maturation. Culturing stem cells under conditions that initiate the myogenic or cardiogenic programs may also prime cells for successful regeneration. Active pursuit of these alternative approaches should be fully investigated as we advance into regenerative medicine.

\section{Methods}

Animals. Sgcd ${ }^{-/-}$mice were previously reported (15). Heterozygous null animals were backcrossed into the C57BL/6J strain through 10 generations. C57BL/6J mice (The Jackson Laboratory) were used as donors for stem cell transplantation. Animals were housed, treated, and handled in accordance with the guidelines set forth by the University of Chicago's Institutional Animal Care and Use Committee, the Animal Welfare Act regulations, and the NIH's Guide for the Care and Use of Laboratory Animals.

Stem cell transplantation. The isolation of SP cells was performed as described by Goodell et al. (6) with modifications. Whole bone marrow was isolated from 6 - to 8 -week-old male mice, and $1 \times 10^{6}$ cells per milliliter were incubated in DMEM (Invitrogen Corp.) with $5 \mu \mathrm{g} / \mathrm{ml}$ Hoechst dye 33342 for 90 minutes at $37^{\circ} \mathrm{C}$ (6). Cells were incubated with a mouse lineage panel of biotinylated antibodies directed against red blood cells and all lymphocytes (BD Biosciences - Pharmingen) at $10^{8}$ cells per milliliter and then incubated with streptavidin-conjugated microbeads (Miltenyi Biotec Inc.) at $4^{\circ} \mathrm{C}$ for 15 minutes. Lineage-positive cells were depleted by manual separation with MidiMACS or automated separation with autoMACS (Miltenyi Biotec Inc.). A subset of cells was stained with a PE-conjugated Sca-1 antibody (BD Biosciences - Pharmingen) for 20 minutes on ice before cell sorting. Propidium iodide was added to the cells immediately prior to cell sorting to exclude dead cells. Lineage-negative cells were subjected to fluorescence-activated cell sorting (FACS; BD
Biosciences), using the MoFlo (DakoCytomation) with the following filter sets: 450/65 bandpass (BP) to detect Hoechst blue, 630/30 BP to detect Hoechst red, and a 560-short pass dichroic filter to separate Hoechst red from Hoechst blue. A subset of cells treated with verapamil confirmed the identity of the SP gate.

Recipient mice were nulliparous $\mathrm{Sgcd}^{-/-}$females, 8-12 weeks of age. Nine of 14 mice were sublethally irradiated with 5 Gy immediately prior to the injection of stem cells. Recipient mice were anesthetized with Avertin and then retro-orbitally injected with $4 \times 10^{3}$ to $30 \times 10^{3}$ (average, $13 \times 10^{3}$ ) BM-SP cells. An additional 2 irradiated mice were retro-orbitally injected with $10 \times 10^{6}$ whole bone marrow cells instead of BM-SP cells. Three weeks or 3 months after transplantation, muscles were dissected and frozen in liquid nitrogen-cooled isopentane as previously described (15).

Direct injection of primary myoblasts or BM-SP cells. Normal primary myoblasts were cultured as previously described (49). Ten $\times 10^{6}$ cells per milliliter in PBS were injected into $\operatorname{Sgcd}^{-/-}$mice $(n=2)$ preirradiated with 5 Gy immediately before injection. A $25-\mu$ l Hamilton Co. syringe was inserted into a longitudinal incision over the tibialis anterior muscle until it penetrated the belly of the muscle. Ten microliters of cells or PBS was delivered to the muscle. Thirty-two days after transplantation, the mice were sacrificed. For direct injection of BM-SP cells, 7,000 cells were injected into the tibialis anterior of $\mathrm{Sgcd} \mathrm{d}^{-/}$mice under direct visualization.

Immunostaining and FISH. Every tenth cryosection of quadriceps or heart was collected on a slide, and 2 serial sections were collected on additional slides. Cryosections of $10 \mu \mathrm{m}$ thickness were processed for IFM with the following antibodies: polyclonal anti- $\gamma$-sarcoglycan $(50)(1: 1,000)$, polyclonal anti- $\delta$-sarcoglycan (15) (1:300), polyclonal anti- $\beta$-sarcoglycan (51) (1:300), polyclonal anti-dystrophin (52) $(1: 1,000)$, or rat monoclonal CD45 (BD Biosciences - Pharmingen) (1:20). Secondary goat anti-rabbit Cy3-conjugated secondary antibody was used for all polyclonal antibodies (Jackson ImmunoResearch Laboratories Inc.). A biotinylated anti-rat IgG
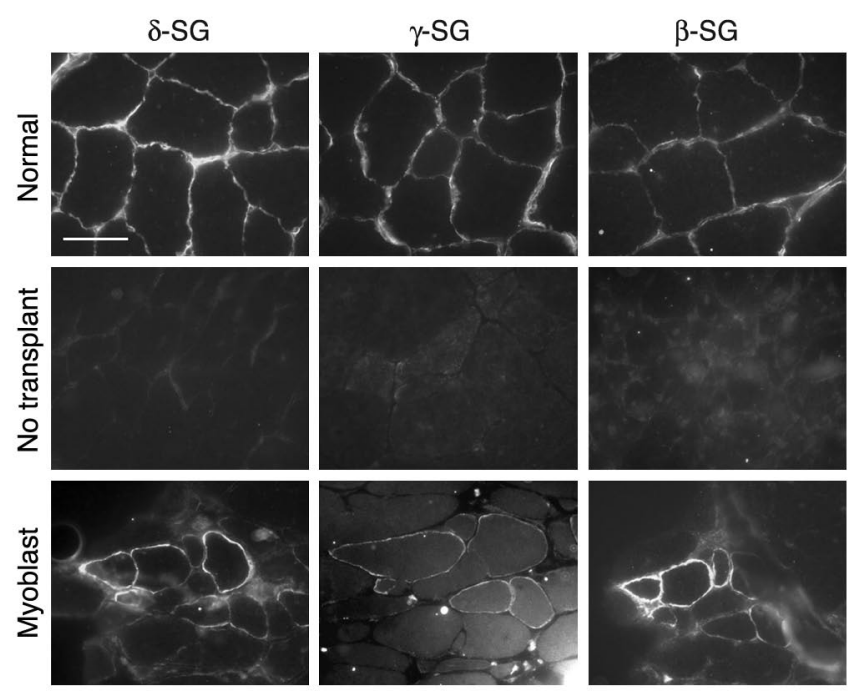

Figure 8

Transplantation of normal primary myoblasts restores the sarcoglycan complex in $\mathrm{Sgcd}^{-1-}$ mice. The top panels represent normal control muscle without transplant. The middle panels are from an $\mathrm{Sgcd}^{-1-}$ mouse. The bottom panels show serial sections from an $\mathrm{Sgcd}^{-1-}$ mouse that underwent direct injection of wild-type myoblasts. Sections were immunostained with $\delta$-sarcoglycan $(\delta-S G), \gamma$-sarcoglycan $(\gamma-S G)$, or $\beta$-sarcoglycan ( $\beta$-SG). Myoblast transplantation can be effective to restore the sarcoglycan complex in $\delta$-sarcoglycan-null muscle. Scale bar: $25 \mu \mathrm{m}$. 
secondary antibody followed by streptavidin-Cy3 was used to detect CD45. Immunostaining was performed alone or followed by FISH.

A Y-1 probe nick-translated with digoxigenin was used to detect the mouse Y chromosome $(21,53)$ (Roche Applied Science). Salmon sperm DNA was added as a blocking agent at a final concentration of $100 \mathrm{ng} / \mu \mathrm{l}$. IFM was combined with FISH as previously described (54), with modifications. Sections from normal male and nontransplanted $\mathrm{Sgcd}^{-/}$- female quadriceps or cardiac tissue were included each time as controls on the same slides as the transplanted tissues. Incubations in primary antibody were overnight at $4^{\circ} \mathrm{C}$, and incubations in secondary antibody were for 1 hour at room temperature. Slides were immediately fixed in HistoChoice MB (AMRESCO Inc.) for 45 minutes. The probe was added, and signal was detected with an anti-digoxigenin antibody conjugated to fluorescein (Roche Applied Science) (1:200). The slides were mounted with VECTASHIELD plus DAPI (Vector Laboratories Inc.).

Analysis of sarcoglycan expression and engraftment. Entire muscle sections were examined for both the expression of $\gamma$-sarcoglycan and the presence of Y chromosome-positive cells. In quadriceps muscle, 198 sections from a total of 5 mice were examined in the 3-week irradiated group, 105 sections from 5 mice were examined in the 3-week nonirradiated group, and 166 sections from 4 mice were examined in the 3 -month irradiated group. In the heart, 124, 124, and 17 sections were analyzed in the 3 groups, respectively. An additional 172 sections from the hearts of the 3-month irradiated group were analyzed for IFM alone with $\gamma$ - or $\delta$-sarcoglycan antibodies. Additional sections in other groups were also immunostained for $\gamma$ - or $\delta$-sarcoglycan without FISH.

A subset of quadriceps muscle was used for quantification of donor cell engraftment. Slides were first immunostained for dystrophin to outline the sarcolemma of the myofibers and then processed for FISH to detect $\mathrm{Y}$ chromosome-positive cells. Images were acquired at regular field intervals throughout 1-2 sections for each mouse in each group as well as in an untransplanted $\mathrm{Sgcd}^{-/-}$mouse. The total area analyzed for each mouse in each group was $0.4-0.5 \mathrm{~mm}^{2}$. An investigator blinded to the different groups quantified the total number of $\mathrm{Y}$ chromosome-positive nuclei present, and, of those, the number that engrafted between myofibers and the number that engrafted inside the myofibers as a central or peripheral nucleus. For cardiac analysis, a total area of $12-58 \mathrm{~mm}^{2}$ per heart $(n=14$; an estimated 4,000 nuclei per square millimeter) was analyzed to quantify the number of Y chromosome-positive nuclei per square millimeter. Over 6,100 Y chromosome-positive nuclei were quantified. A subset of cardiac sections (1-2 heart sections per heart) was immunostained for dystrophin followed by FISH with the Y-1 probe. Over 2,000 Y chromosome-positive nuclei were detected and scored for their localization between or within cardiomyocytes.

Histology. Quadriceps and heart were sectioned and processed for Masson trichrome staining.

Microscopy. Fluorescent images were acquired using an Axioskop, AxioCam, and AxioVision (Carl Zeiss Inc.). An SP2 AOBS laser scanning confocal microscope (Leica Microsystems Inc.) was used to collect confocal images.

Statistical analysis. Data were calculated using Microsoft Excel (Microsoft Corp.) and are presented as mean \pm SD. Nonparametric statistics were calculated with GraphPad Prism (GraphPad Software Inc.). All values given are 2 -tailed $P$ values.

\section{Acknowledgments}

E.M. McNally is supported by the Muscular Dystrophy Association, the NIH, the American Heart Association, and the Burroughs Wellcome Fund. K.A. Lapidos is supported by an American Heart Association Predoctoral Fellowship (Midwest Affiliate). A. Ma is supported by the NIH.

Received for publication August 18, 2004, and accepted in revised form September 28, 2004.

Address correspondence to: Elizabeth M. McNally, The University of Chicago, 5841 South Maryland, MC6088, Chicago, Illinois 60637, USA. Phone: (773) 702-2672; Fax: (773) 702-2681; E-mail: emcnally@medicine.bsd.uchicago.edu.
1. Frisen, J. 2002. Stem cell plasticity? Neuron. 35:415-418.

2. Morrison, S.J. 2001. Stem cell potential: can anything make anything? Curr. Biol. 11:R7-R9.

3. Corti, S., et al. 2002. A subpopulation of murine bone marrow cells fully differentiates along the myogenic pathway and participates in muscle repair in the $\mathrm{mdx}$ dystrophic mouse. Exp. Cell Res. 277:74-85.

4. LaBarge, M.A., and Blau, H.M. 2002. Biological progression from adult bone marrow to mononucleate muscle stem cell to multinucleate muscle fiber in response to injury. Cell. 111:589-601.

5. Camargo, F.D., Green, R., Capetenaki, Y., Jackson, K.A., and Goodell, M.A. 2003. Single hematopoietic stem cells generate skeletal muscle through myeloid intermediates. Nat. Med. 9:1520-1527.

6. Goodell, M.A., Brose, K., Paradis, G., Conner, A.S., and Mulligan, R.C. 1996. Isolation and functional properties of murine hematopoietic stem cells that are replicating in vivo. J. Exp. Med. 183:1797-1806.

7. Gussoni, E., et al. 1999. Dystrophin expression in the mdx mouse restored by stem cell transplantation. Nature. 401:390-394.

8. Jackson, K.A., et al. 2001. Regeneration of ischemic cardiac muscle and vascular endothelium by adult stem cells. J. Clin. Invest. 107:1395-1402.

9. Montanaro, F., Liadaki, K., Volinski, J., Flint, A., and Kunkel, L.M. 2003. Skeletal muscle engraftment potential of adult mouse skin side population cells. Proc. Natl. Acad. Sci. U. S. A. 100:9336-9341.

10. Welm, B.E., et al. 2002. Sca-1(pos) cells in the mouse mammary gland represent an enriched pro- genitor cell population. Dev. Biol. 245:42-56.

11. Hierlihy, A.M., Seale, P., Lobe, C.G., Rudnicki, M.A., and Megeney, L.A. 2002. The post-natal heart contains a myocardial stem cell population. FEBS Lett. 530:239-243.

12. Orlic, D., et al. 2001. Bone marrow cells regenerate infarcted myocardium. Nature. 410:701-705.

13. Murry, C.E., et al. 2004. Haematopoietic stem cells do not transdifferentiate into cardiac myocytes in myocardial infarcts. Nature. 428:664-668.

14. Bittner, R.E., et al. 1999. Recruitment of bone-marrow-derived cells by skeletal and cardiac muscle in adult dystrophic mdx mice. Anat. Embryol. 199:391-396.

15. Hack, A.A., et al. 2000. Differential requirement for individual sarcoglycans and dystrophin in the assembly and function of the dystrophinglycoprotein complex. J. Cell Sci. 113:2535-2544.

16. Lynch, G.S., Rafael, J.A., Chamberlain, J.S., and Faulkner, J.A. 2000. Contraction-induced injury to single permeabilized muscle fibers from $\mathrm{mdx}$, transgenic mdx, and control mice. Am. J. Physiol. Cell Physiol. 279:C1290-C1294.

17. Coral-Vazquez, R., et al. 1999. Disruption of the sarcoglycan-sarcospan complex in vascular smooth muscle: a novel mechanism for cardiomyopathy and muscular dystrophy. Cell. 98:465-474.

18. Factor, S.M., Minase, T., Cho, S., Dominitz, R., and Sonnenblick, E.H. 1982. Microvascular spasm in the cardiomyopathic Syrian hamster: a preventable cause of focal myocardial necrosis. Circulation. 66:342-354.

19. Corbel, S.Y., et al. 2003. Contribution of hemato- poietic stem cells to skeletal muscle. Nat. Med. 9:1528-1532.

20. Torrente, Y., et al. 2001. Intraarterial injection of muscle-derived CD34(+)Sca-1(+) stem cells restores dystrophin in mdx mice. J. Cell Biol. 152:335-348.

21. Irintchev, A., Langer, M., Zweyer, M., Theisen, R., and Wernig, A. 1997. Functional improvement of damaged adult mouse muscle by implantation of primary myoblasts. J. Physiol. 500:775-785.

22. Cordier, L., et al. 2001. Muscle-specific promoters may be necessary for adeno-associated virus-mediated gene transfer in the treatment of muscular dystrophies. Hum. Gene Ther. 12:205-215.

23. Greelish, J.P., et al. 1999. Stable restoration of the sarcoglycan complex in dystrophic muscle perfused with histamine and a recombinant adeno-associated viral vector. Nat. Med. 5:439-443.

24. Li, J., et al. 1999. rAAV vector-mediated sarcogylcan gene transfer in a hamster model for limb girdle muscular dystrophy. Gene Ther. 6:74-82.

25. Wheeler, M.T., et al. 2004. Smooth muscle cell-extrinsic vascular spasm arises from cardiomyocyte degeneration in sarcoglycan-deficient cardiomyopathy. J. Clin. Invest. 113:668-675. doi:10.1172/JCI200420410.

26. Beauchamp, J.R., Morgan, J.E., Pagel, C.N., and Partridge, T.A. 1999. Dynamics of myoblast transplantation reveal a discrete minority of precursors with stem cell-like properties as the myogenic source. J. Cell Biol. 144:1113-1122.

27. Morgan, J.E., Pagel, C.N., Sherratt, T., and Partridge, T.A. 1993. Long-term persistence and migration of myogenic cells injected into pre-irradiated muscles 
of mdx mice. J. Neurol. Sci. 115:191-200.

28. Porter, J.D., et al. 2002. A chronic inflammatory response dominates the skeletal muscle molecular signature in dystrophin-deficient mdx mice. Hum. Mol. Genet. 11:263-272.

29. Orlic, D., et al. 2001. Mobilized bone marrow cells repair the infarcted heart, improving function and survival. Proc. Natl. Acad. Sci. U. S. A. 98:10344-10349.

30. Balsam, L.B., et al. 2004. Haematopoietic stem cells adopt mature haematopoietic fates in ischaemic myocardium. Nature. 428:668-673.

31. Bittmann, I., Hentrich, M., Bise, K., Kolb, H.J., and Lohrs, U. 2003. Endothelial cells but not epithelial cells or cardiomyocytes are partially replaced by donor cells after allogeneic bone marrow and stem cell transplantation. J. Hematother. Stem Cell Res. 12:359-366

32. Deb, A., et al. 2003. Bone marrow-derived cardiomyocytes are present in adult human heart: a study of gender-mismatched bone marrow transplantation patients. Circulation. 107:1247-1249.

33. Glaser, R., Lu, M.M., Narula, N., and Epstein, J.A. 2002. Smooth muscle cells, but not myocytes, of host origin in transplanted human hearts. Circulation. 106:17-19.

34. Laflamme, M.A., Myerson, D., Saffitz, J.E., and Murry, C.E. 2002. Evidence for cardiomyocyte repopulation by extracardiac progenitors in transplanted human hearts. Circ. Res. 90:634-640.

35. Strauer, B.E., et al. 2002. Repair of infarcted myocardium by autologous intracoronary mononuclear bone marrow cell transplantation in humans. Circulation. 106:1913-1918.

36. Perin, E.C., et al. 2003. Transendocardial, autologous bone marrow cell transplantation for severe, chronic ischemic heart failure. Circulation. 107:2294-2302.

37. Assmus, B., et al. 2002. Transplantation of Progenitor Cells and Regeneration Enhancement in Acute Myocardial Infarction (TOPCARE-AMI). Circulation. 106:3009-3017.

38. Lee, M.S., and Makkar, R.R. 2004. Stem-cell transplantation in myocardial infarction: a status report. Ann. Intern. Med. 140:729-737.

39. Noguchi, S., Wakabayashi, E., Imamura, M., Yoshida, M., and Ozawa, E. 1999. Developmental expression of sarcoglycan gene products in cultured myocytes. Biochem. Biophys. Res. Commun. 262:88-93.

40. Sampaolesi, M., et al. 2003. Cell therapy of alphasarcoglycan null dystrophic mice through intraarterial delivery of mesoangioblasts. Science. 301:487-492.

41. Gussoni, E., et al. 2002. Long-term persistence of donor nuclei in a Duchenne muscular dystrophy patient receiving bone marrow transplantation. J. Clin. Invest. 110:807-814. doi:10.1172/ JCI200216098.

42. Wagers, A.J., Sherwood, R.I., Christensen, J.L., and Weissman, I.L. 2002. Little evidence for developmental plasticity of adult hematopoietic stem cells. Science. 297:2256-2259.

43. Castro, R.F., et al. 2002. Failure of bone marrow cells to transdifferentiate into neural cells in vivo. Science. 297:1299.

44. Ferrari, G., Stornaiuolo, A., and Mavilio, F. 2001. Failure to correct murine muscular dystrophy. Nature. 411:1014-1015.

45. Ono, K., et al. 2003. Preservation of hematopoietic properties in transplanted bone marrow cells in the brain. J. Neurosci. Res. 72:503-507.

46. Alvarez-Dolado, M., et al. 2003. Fusion of bone-marrow-derived cells with Purkinje neurons, cardiomyocytes and hepatocytes. Nature. 425:968-973.

47. Oh, H., et al. 2003. Cardiac progenitor cells from adult myocardium: homing, differentiation, and fusion after infarction. Proc. Natl. Acad. Sci. U. S. A. 100:12313-12318.

48. Qu-Petersen, Z., et al. 2002. Identification of a novel population of muscle stem cells in mice: potential for muscle regeneration. J. Cell Biol. 157:851-864.

49. Rando, T.A., and Blau, H.M. 1994. Primary mouse myoblast purification, characterization, and transplantation for cell-mediated gene therapy. J. Cell Biol. 125:1275-1287.

50. McNally, E.M., et al. 1996. Mutations that disrupt the carboxyl-terminus of gamma-sarcoglycan cause muscular dystrophy. Hum. Mol. Genet. 5:1841-1847.

51. Bonnemann, C.G., et al. 1996. Genomic screening for beta-sarcoglycan gene mutations: missense mutations may cause severe limb-girdle muscular dystrophy type 2E (LGMD 2E). Hum. Mol. Genet. 5:1953-1961.

52. Lidov, H.G., Byers, T.J., Watkins, S.C., and Kunkel, L.M. 1990. Localization of dystrophin to postsynaptic regions of central nervous system cortical neurons. Nature. 348:725-728.

53. Nishioka, Y. 1988. Application of Y chromosomal repetitive sequences to sexing mouse embryos. Teratology. 38:181-185.

54. Gussoni, E., et al. 1996. A method to codetect introduced genes and their products in gene therapy protocols. Nat. Biotechnol. 14:1012-1016. 